\title{
Integral Homology of the Moduli Space of Tropical Curves of Genus 1 with Marked Points
}

\author{
Ye LiU
}

(Received January 15, 2014; Revised December 11, 2014)

\begin{abstract}
Kozlov has studied the topological properties of the moduli space of tropical curves of genus 1 with marked points, such as its mod 2 homology, while the integral homology remained a conjecture. In this paper, we present a complete proof of Kozlov's conjecture concerning the integral homology of this moduli space.
\end{abstract}

Key words: tropical curve, moduli space, equivariant homology.

\section{Introduction}

Tropical curves are objects of interest in the field of tropical geometry. The moduli spaces of tropical curves with marked points were introduced by Mikhalkin in [6], [7] from the tropical geometric point of view. Kozlov, on the other hand, has investigated the same object from the topological point of view in [3], [4], [5]. In particular, he studied the genus 1 case in depth. Among other things, Kozlov showed the following property.

Theorem 1.1 (Kozlov) Let $n$ be a positive integer, then the moduli space $T M_{1, n+1}$ of tropical curves of genus 1 with $n+1$ marked points is homotopy equivalent to a quotient space $T^{n} / \mathbb{Z}_{2}$ of the $n$-torus, where $\mathbb{Z}_{2}$ acts diagonally on $T^{n}=S^{1} \times \cdots \times S^{1}$ by conjugation of each factor $S^{1}$ viewed as the unit circle of the complex plane. Therefore

$$
H_{*}\left(T M_{1, n+1}\right) \cong H_{*}\left(T^{n} / \mathbb{Z}_{2}\right)
$$

Using Theorem 1.1, Kozlov computed the mod 2 homology of $T M_{1, n+1}$. His result is as follows.

Theorem 1.2 (Kozlov) The mod 2 homology of $T M_{1, n+1}$ has the form

$$
\tilde{H}_{k}\left(T M_{1, n+1} ; \mathbb{Z}_{2}\right) \cong \mathbb{Z}_{2}^{\tilde{\beta}_{k}\left(T^{n} / \mathbb{Z}_{2} ; \mathbb{Z}_{2}\right)}
$$


where

$$
\tilde{\beta}_{k}\left(T^{n} / \mathbb{Z}_{2} ; \mathbb{Z}_{2}\right)= \begin{cases}\left(\begin{array}{l}
n-1 \\
k-1
\end{array}\right)+2\left(\begin{array}{l}
n-2 \\
k-1
\end{array}\right)+\cdots+2^{n-k}\left(\begin{array}{l}
k-1 \\
k-1
\end{array}\right), & 2 \leq k \leq n \\
0, & \text { otherwise }\end{cases}
$$

Kozlov also suggested a conjecture concerning the integral homology of $T M_{1, n+1}$. Our main result is a proof of his conjecture.

Theorem 1.3 The integral homology of the moduli space $T M_{1, n+1}$ of tropical curves of genus 1 with $n+1$ marked points has the form

$$
\begin{array}{rlrl}
\tilde{H}_{2 i}\left(T M_{1, n+1}\right) \cong \mathbb{Z}_{2}^{a(i, n)} \oplus \mathbb{Z}^{b(i, n)}, & & 2 \leq 2 i \leq n, \\
\tilde{H}_{j}\left(T M_{1, n+1}\right) & =0, & & \text { otherwise. }
\end{array}
$$

where

$$
\begin{aligned}
a(i, n) & =\tilde{\beta}_{2 i+1}\left(T^{n} / \mathbb{Z}_{2} ; \mathbb{Z}_{2}\right), \\
a(i, n)+b(i, n) & =\tilde{\beta}_{2 i}\left(T^{n} / \mathbb{Z}_{2} ; \mathbb{Z}_{2}\right) .
\end{aligned}
$$

In Section 2, we present the definition of the space $T M_{1, n+1}$ in study and explain Theorem 1.1. Then we focus on the space $T^{n} / \mathbb{Z}_{2}$. Section 3 consists of a description of a cellular structure of $T^{n} / \mathbb{Z}_{2}$ that is suitable for our computation. In order to conclude our main theorem from the result of the mod 2 homology and the universal coefficient theorem, it suffices to show the following two claims.

- The homology group $H_{2 i+1}\left(T^{n} / \mathbb{Z}_{2}\right)$ is trivial for all $i$. (Proposition 5.1)

- The homology group $H_{2 i}\left(T^{n} / \mathbb{Z}_{2}\right)$ has no odd torsion nor higher 2torsion for $2 \leq 2 i \leq n$. (Proposition 5.2)

Section 4 and 5 are devoted to proving the two claims.

Throughout this paper, homology means integral homology unless otherwise specified, and $\tilde{H}$ means reduced homology. 


\section{The moduli space of metric graphs of genus 1 with marked points.}

Kozlov studied the topological properties of the moduli spaces of tropical curves with marked points in [3], [4], [5]. The contents of this section are taken from [4]. However our definitions and notations here are slightly different.

Definition 2.1 A finite graph $G$ (allowing loops and multiedges) is called a metric graph if it is given an edge-length function

$$
l_{G}: E(G) \rightarrow(0, \infty),
$$

where $E(G)$ denotes the set of edges of $G$. For a nonnegative integer $n$, a metric graph $G$ is called a metric graph with $n$ marked points if it is given a marking function

$$
p_{G}:[n] \rightarrow \Delta(G)
$$

where $[n]:=\{1, \ldots, n\}$ for $n \geq 1$ and $[0]:=\emptyset, \Delta(G)$ is the space obtained by viewing $G$ as a 1-dimensional CW complex.

Let $M G_{n}$ denote the set of isometry classes of finite metric graphs with $n$ marked points. Kozlov introduced a suitable topology for $M G_{n}$ and called the obtained topological space the moduli space of metric graphs with $n$ marked points. Here we describe this topology in brief. The interested reader is referred to Subsection 3.1 of [4] for an explicit definition.

Let $G$ be a metric graph with $n$ marked points. Set $r(G):=\min d(x, y)$, where $x, y$ run over the set of vertices and marked points, $d$ is the standard metric on $\Delta(G)$ induced by $l_{G}$. (The explicit definition of this metric is given in Subsection 2.3 of [4].) Now for a number $\varepsilon \in(0, r(G) / 2)$, we define a set $N_{\varepsilon}(G)$ by saying that a metric graph $H$ with $n$ marked points is in $N_{\varepsilon}(G)$ if and only if

- the edges of $H$ of lengths less than $\varepsilon$ form a subforest;

- the graph $G$ can be obtained from $H$ by first shrinking all the edges of lengths less than $\varepsilon$ and then varying the lengths of the remaining edges and positions of marked points by up to $\varepsilon$.

For an isometry class $[G]$, we set $N_{\varepsilon}([G]):=\left\{[H] \mid H \in N_{\varepsilon}(G)\right\}$. This is 
independent of the choice of representatives. The topology of $M G_{n}$ can be given as follows: a subset $X \subset M G_{n}$ is open if and only if for every $[G] \in X$, there exists $\varepsilon \in(0, r(G) / 2)$ such that $N_{\varepsilon}([G]) \subset X$.

Definition 2.2 Let $d$ be a positive real number. We define $T M_{n}(d)$ to be the subspace of $M G_{n}$ consisting of the isometry classes of all connected metric graphs $G$ with $n$ marked points, such that

- $G$ has no vertices of valency 2 ;

- $G$ has exactly $n$ leaves (vertices of valency 1 ), and these are marked 1 through $n$;

- the lengths of the edges leading to leaves are equal to $d$.

Note that for arbitrary $d_{1}, d_{2} \in(0, \infty), T M_{n}\left(d_{1}\right)$ and $T M_{n}\left(d_{2}\right)$ are homeomorphic. So we could suppress $d$ and just write $T M_{n}$. Furthermore $T M_{n}$ is homeomorphic to the moduli space of tropical curves with $n$ marked points. The latter can be "considered" as $T M_{n}(\infty)$. See Section 3.5 of [4] for details.

Recall that the genus of a graph $G$ is the first Betti number of $\Delta(G)$. It is known that the connected components of $T M_{n}$ are indexed by the genera of consisting graphs. We denote by $T M_{g, n}$ the connected component of $T M_{n}$ consisting of isometry classes of graphs of genus $g$. Remark that $T M_{g, n}$ is homeomorphic to the moduli space of tropical curves of genus $g$ with $n$ marked points.

For the case $g=1, T M_{1, n+1} \simeq S^{1} \times \cdots \times S^{1} / O(2)=T^{n+1} / O(2)$, where $O(2)$ acts on each $S^{1}$ as orthogonal transformation and diagonally on $T^{n+1}$. We see that $T^{n+1} / O(2)$ is homeomorphic to $T^{n} / \mathbb{Z}_{2}$ by fixing the last coordinate of a point on $T^{n+1}$ to be $1 \in \mathbb{C}$. Hence we conclude Theorem 1.1 (See Section 4.1 of [4]).

Theorem 2.3 (Kozlov) We have the following homotopy equivalence,

$$
T M_{1, n+1} \simeq T^{n} / \mathbb{Z}_{2},
$$

where the nonidentity $t$ of $\mathbb{Z}_{2}$ acts on $T^{n}=S^{1} \times \cdots \times S^{1}$ as

$$
t\left(z_{1}, \ldots, z_{n}\right)=\left(\bar{z}_{1}, \ldots, \bar{z}_{n}\right)
$$

for $z_{1}, \ldots, z_{n}$ on the unit circle of the complex plane. Therefore, 


$$
H_{*}\left(T M_{1, n+1}\right) \cong H_{*}\left(T^{n} / \mathbb{Z}_{2}\right)
$$

From now on, we focus on the computation of $H_{*}\left(T^{n} / \mathbb{Z}_{2}\right)$.

\section{Cellular structures}

We shall give $T^{n} / \mathbb{Z}_{2}$ a cellular structure so that we could compute $H_{*}\left(T^{n} / \mathbb{Z}_{2}\right)$. First let us give a cellular structure to the unit circle $S^{1}$ of $\mathbb{C}$ as follows.

- 0-cells: $e_{0}^{+}, e_{0}^{-}$denoting $1,-1 \in S^{1}$ respectively.

- 1-cells: $e_{1}^{+}, e_{1}^{-}$denoting the upper and lower arcs joining -1 with 1 respectively with orientations given by the following boundary maps.

- boudary maps: $\partial e_{1}^{+}=\partial e_{1}^{-}=e_{0}^{+}-e_{0}^{-}$.

Thus $T^{n}=S^{1} \times \cdots \times S^{1}$ has been given a cellular structure as follows.

- $k$-cells $(0 \leq k \leq n): a_{1} \times \cdots \times a_{n}$

where $a_{i_{l}} \in\left\{e_{1}^{+}, e_{1}^{-}\right\}$for $l=1,2, \ldots, k$ with $1 \leq i_{1}<i_{2}<\cdots<i_{k} \leq$ $n$ and

$a_{j} \in\left\{e_{0}^{+}, e_{0}^{-}\right\}$for other $j$.

- boundary maps:

$$
\partial\left(a_{1} \times \cdots \times a_{n}\right)=\sum_{i=1}^{n}(-1)^{d_{0}+d_{1}+\cdots+d_{i-1}} a_{1} \times \cdots \times \partial a_{i} \times \cdots \times a_{n}
$$

where $d_{l}=\operatorname{dim} a_{l}$ for $l=1,2, \ldots, n-1$ and $d_{0}=0$. In particular, for $a_{1} \times \cdots \times a_{n}$ as above,

$$
\partial\left(a_{1} \times \cdots \times a_{n}\right)=\sum_{l=1}^{k}(-1)^{l-1} a_{1} \times \cdots \times \partial a_{i_{l}} \times \cdots \times a_{n}
$$

where $\partial a_{i_{l}}=e_{0}^{+}-e_{0}^{-}$.

Now consider $\mathbb{Z}_{2}=\{e, t\}$ acting on $S^{1}$ by

- $e\left(e_{0}^{ \pm}\right)=e_{0}^{ \pm}, e\left(e_{1}^{ \pm}\right)=e_{1}^{ \pm}$.

- $t\left(e_{0}^{ \pm}\right)=e_{0}^{ \pm}, t\left(e_{1}^{ \pm}\right)=e_{1}^{\mp}$.

The group $\mathbb{Z}_{2}$ acts on $T^{n}$ diagonally, therefore we obtain an induced cellular structure of $T^{n} / \mathbb{Z}_{2}$. 
- $k$-cells: $\overline{a_{1} \times \cdots \times a_{n}}$, where $a_{1} \times \cdots \times a_{n}$ is a $k$-cell of $T^{n}$ as above and overline means $\mathbb{Z}_{2}$-orbit. Note that $\overline{a_{1} \times \cdots \times a_{n}}=\overline{t\left(a_{1} \times \cdots \times a_{n}\right)}$.

- Induced boundary maps:

$$
\begin{aligned}
& \bar{\partial} \overline{a_{1} \times \cdots \times a_{n}} \\
& \left.\quad=\sum_{l=1}^{k}(-1)^{l-1} \overline{\left(\overline{a_{1} \times \cdots \times e_{0}^{+} \times \cdots \times a_{n}}\right.}-\overline{a_{1} \times \cdots \times e_{0}^{-} \times \cdots \times a_{n}}\right) .
\end{aligned}
$$

Let us denote the complement of $\left\{i_{1}, \cdots, i_{k}\right\}$ in $[n]$ by $\left\{j_{1}, \ldots, j_{m}\right\}$ with $j_{1}<\cdots<j_{m}(k+m=n)$. Then we introduce the following sets.

$$
\begin{aligned}
& A\left(i_{1}, \ldots, i_{k}\right) \\
& \quad=\left\{\overline{a_{1} \times \cdots \times a_{n}} \mid a_{i_{1}}=\cdots=a_{i_{k}}=e_{1}^{+}, a_{j_{1}}, \cdots, a_{j_{m}} \in\left\{e_{0}^{+}, e_{0}^{-}\right\}\right\},
\end{aligned}
$$

and

$$
B\left(i_{1}, \ldots, i_{k} ; j_{l}\right)=\left\{\begin{array}{l|l}
\overline{a_{1} \times \cdots \times a_{n}} \mid \begin{array}{l}
a_{i_{1}}=\cdots=a_{i_{k}}=e_{1}^{+}, a_{j_{l}} \in\left\{e_{1}^{+}, e_{1}^{-}\right\} \\
a_{j_{1}}, \ldots, a_{j_{l-1}}, a_{j_{l+1}}, \ldots, a_{j_{m}} \in\left\{e_{0}^{+}, e_{0}^{-}\right.
\end{array}
\end{array}\right\} .
$$

For later use, we prove the following proposition.

Proposition 3.1 Let $k$ be a natural number. For $1 \leq i_{1}<i_{2}<\cdots<$ $i_{k} \leq n$, any $k$-chain $c$ of $T^{n} / \mathbb{Z}_{2}$ can be expressed by

$$
\begin{aligned}
& c=\sum_{\overline{a_{1} \times \cdots \times a_{n}} \in A\left(i_{1}, \ldots, i_{k}\right)} x\left(\overline{a_{1} \times \cdots \times a_{n}}\right) \overline{a_{1} \times \cdots \times a_{n}} \\
& +\sum_{\overline{a_{1} \times \cdots \times a_{n}} \notin A\left(i_{1}, \ldots, i_{k}\right)} x\left(\overline{a_{1} \times \cdots \times a_{n}}\right) \overline{a_{1} \times \cdots \times a_{n}},
\end{aligned}
$$

where $x\left(\overline{a_{1} \times \cdots \times a_{n}}\right) \in \mathbb{Z}$. If $c$ is a $k$-boundary, then

$$
\sum_{\overline{a_{1} \times \cdots \times a_{n}} \in A\left(i_{1}, \ldots, i_{k}\right)} x\left(\overline{a_{1} \times \cdots \times a_{n}}\right)=0 .
$$

Proof. Since $(k+1)$-cells outside of 


$$
\bigcup_{l=1}^{m} B\left(i_{1}, \ldots, i_{k} ; j_{l}\right)
$$

do not have cells in $A\left(i_{1}, \ldots, i_{k}\right)$ as faces, it suffices to compute the boundaries of cells in $B\left(i_{1}, \ldots, i_{k} ; j_{l}\right)$. Suppose $\overline{a_{1} \times \cdots \times a_{n}} \in B\left(i_{1}, \ldots, i_{k} ; j_{l}\right)$, then

$$
\begin{aligned}
\bar{\partial} & \overline{a_{1} \times \cdots \times a_{n}} \\
= & \left.(-1)^{\#\left\{p \mid i_{p}<j_{l}\right\}} \overline{\left(\overline{a_{1} \times \cdots \times e_{0}^{+} \times \cdots \times a_{n}}\right.}-\overline{a_{1} \times \cdots \times e_{0}^{-} \times \cdots \times a_{n}}\right) \\
& +\cdots
\end{aligned}
$$

where the omitted term is a linear combination of cells outside of $A\left(i_{1}, \ldots, i_{k}\right)$. Hence the desired result follows.

\section{Further explorations}

By exactly the same argument as in Section 4.6 of [4], we have the following exact sequence.

$$
\begin{aligned}
\cdots \rightarrow \tilde{H}_{k}\left(T^{n}\right) \stackrel{\left(q_{*}, q_{*}\right)}{\longrightarrow} \tilde{H}_{k}\left(T^{n} / \mathbb{Z}_{2}\right) \oplus \tilde{H}_{k}\left(T^{n} / \mathbb{Z}_{2}\right) & \rightarrow \tilde{H}_{k}\left(T^{n+1} / \mathbb{Z}_{2}\right) \\
& \rightarrow \tilde{H}_{k-1}\left(T^{n}\right) \stackrel{\left(q_{*}, q_{*}\right)}{\longrightarrow} \cdots
\end{aligned}
$$

where $q_{*}: \tilde{H}_{*}\left(T^{n}\right) \rightarrow \tilde{H}_{*}\left(T^{n} / \mathbb{Z}_{2}\right)$ is induced by the quotient map $q: T^{n} \rightarrow$ $T^{n} / \mathbb{Z}_{2}$. We denote the induced chain map by $q_{\#}: C_{*}\left(T^{n}\right) \rightarrow C_{*}\left(T^{n} / \mathbb{Z}_{2}\right)$.

Study of $q_{*}$ requires a detailed discussion on $H_{*}\left(T^{n}\right)$. For $1 \leq i_{1}<$ $\cdots<i_{k} \leq n$, define a $k$-chain $\sigma_{i_{1}, \ldots, i_{k}}$ of $T^{n}$ by

$$
\sigma_{i_{1}, \ldots, i_{k}}=c_{1} \times \cdots \times c_{n} \in C_{k}\left(T^{n}\right),
$$

where

- $c_{i_{l}}=e_{1}^{+}-e_{1}^{-}$for $l=1, \ldots, k$.

- $c_{j}=e_{0}^{+}$for other $j$.

Theorem 4.1 The chain $\sigma_{i_{1}, \ldots, i_{k}}$ is a $k$-cycle of $T^{n}$. Futhermore $H_{k}\left(T^{n}\right)$ is free with basis $\left\{\left[\sigma_{i_{1}, \ldots, i_{k}}\right] \mid 1 \leq i_{1}<\cdots<i_{k} \leq n\right\}$. 
Proof. It is immediate from Künneth formula.

Remark 4.2 The induced $\mathbb{Z}_{2}$-action on $C_{k}\left(T^{n}\right)$ is given by

$$
\begin{gathered}
e\left(\sum \lambda_{i} \tau_{i}\right)=\sum \lambda_{i} e\left(\tau_{i}\right)=\sum \lambda_{i} \tau_{i}, \\
t\left(\sum \lambda_{i} \tau_{i}\right)=\sum \lambda_{i} t\left(\tau_{i}\right) .
\end{gathered}
$$

Then we conclude

$$
\begin{aligned}
q_{\#}\left(t\left(\sum \lambda_{i} \tau_{i}\right)\right) & =q_{\#}\left(\sum \lambda_{i} t\left(\tau_{i}\right)\right)=\sum \lambda_{i} \overline{t\left(\tau_{i}\right)} \\
& =\sum \lambda_{i} \overline{\tau_{i}}=q_{\#}\left(\sum \lambda_{i} \tau_{i}\right) .
\end{aligned}
$$

Proposition 4.3 The induced homomorphism

$$
q_{*}: H_{k}\left(T^{n}\right) \rightarrow H_{k}\left(T^{n} / \mathbb{Z}_{2}\right)
$$

is the 0-map if $k$ is odd and is injective if $k$ is even.

Proof. It suffices to investigate $q_{*}$ applied to the basis.

$$
\begin{aligned}
& q_{*}\left(\left[\sigma_{i_{1}, \ldots, i_{k}}\right]\right)=\left[q_{\#}\left(\sigma_{i_{1}, \ldots, i_{k}}\right)\right] \\
& =\left[q_{\#}\left(c_{1} \times \cdots \times e_{i_{1}}^{+} \times \cdots \times c_{n}\right)-q_{\#}\left(c_{1} \times \cdots \times e_{i_{1}}^{-} \times \cdots \times c_{n}\right)\right] \\
& =\left[q_{\#}\left(c_{1} \times \cdots \times e_{i_{1}}^{+} \times \cdots \times c_{n}\right)\right. \\
& +(-1)^{k} q_{\#}\left(c_{1} \times \cdots \times e_{i_{1}}^{-} \times \cdots \times\left(-c_{i_{2}}\right)\right. \\
& \left.\left.\times \cdots \times\left(-c_{i_{k}}\right) \times \cdots \times c_{n}\right)\right] \\
& =\left[q_{\#}\left(c_{1} \times \cdots \times e_{1}^{+} \times \cdots \times c_{n}\right)\right. \\
& \left.+(-1)^{k} q_{\#}\left(t\left(c_{1} \times \cdots \times e_{i_{1}}^{+} \times \cdots \times c_{n}\right)\right)\right] \\
& = \begin{cases}0, & k: \text { odd } ; \\
{\left[2 q_{\#}\left(c_{1} \times \cdots \times e_{1}^{+} \times \cdots \times c_{n}\right)\right],} & k: \text { even. }\end{cases}
\end{aligned}
$$


Thus $q_{*}$ is the 0 -map if $k$ is odd. To see the injectivity when $k$ is even, take a chain

$$
\sigma=\sum_{1 \leq i_{1}<\cdots<i_{k} \leq n} x_{i_{1}, \ldots, i_{k}} \sigma_{i_{1}, \ldots, i_{k}} \in C_{k}\left(T^{n}\right)
$$

such that

$$
q_{\#}(\sigma)=q_{\#}\left(\sum x_{i_{1}, \ldots, i_{k}} \sigma_{i_{1}, \ldots, i_{k}}\right)
$$

is a $k$-boundary of $T^{n} / \mathbb{Z}_{2}$. We show that all the coefficients $x_{i_{1}, \ldots, i_{k}}$ 's are 0 . We have already obtained

$q_{\#}(\sigma)=\sum x_{i_{1}, \ldots, i_{k}} q_{\#}\left(\sigma_{i_{1}, \ldots, i_{k}}\right)=\sum 2 x_{i_{1}, \ldots, i_{k}} q_{\#}\left(c_{1} \times \cdots \times e_{i_{1}}^{+} \times \cdots \times c_{n}\right)$,

where $q_{\#}\left(c_{1} \times \cdots \times e_{1}^{+} \times \cdots \times c_{n}\right)$ is of the form

$$
\sum \pm \overline{e_{0}^{+} \times \cdots \times e_{1}^{+} \times \cdots \times e_{i_{1}}^{ \pm} \times \cdots \times e_{0}^{+}}
$$

which is a linear combination of $2^{k-1} k$-cells, with each cell having a coefficient 1 (resp. -1 ) if it contains even (resp. odd) number of $e_{1}^{-}$'s in its representative with the $i_{1}$-th component $e_{1}^{+}$.

Note that the $k$-cell

$$
\alpha_{i_{1}, \ldots, i_{k}}=\overline{\cdots \times e_{i_{p}}^{+} \times \cdots \times e_{0}^{+} \times \cdots} \in A\left(i_{1}, \ldots, i_{k}\right)
$$

with all $j_{l}$-th components $e_{0}^{+}$is in $(*)$. Then $q_{\#}(\sigma)$ is of the form

$$
q_{\#}(\sigma)=2 x_{i_{1}, \ldots, i_{k}} \alpha_{i_{1}, \ldots, i_{k}}+\sum_{\beta \in A\left(i_{1}, \ldots, i_{k}\right)-\left\{\alpha_{i_{1}, \ldots, i_{k}}\right\}} y_{\beta} \beta+\cdots
$$

where the omitted term is a linear combination of cells outside of $A_{i_{1}, \ldots, i_{k}}$. Since $q_{\#}(\sigma)$ is a $k$-boundary of $T^{n} / \mathbb{Z}_{2}$, by Proposition 3.1,

$$
2 x_{i_{1}, \ldots, i_{k}}+\sum y_{\beta}=0 \text {. }
$$


One also observes that $q_{\#}(\sigma)$ does not contain cells with $e_{0}^{-}$, in particular $\beta$ as above. Thus

$$
y_{\beta}=0
$$

for all $\beta \in A\left(i_{1}, \ldots, i_{k}\right)-\left\{\alpha_{i_{1}, \ldots, i_{k}}\right\}$ and we conclude

$$
x_{i_{1}, \ldots, i_{k}}=0
$$

This completes the proof.

\section{The integral homology}

We are ready to prove the two claims mentioned in the introduction.

Proposition 5.1 The homology $H_{2 i+1}\left(T^{n+1} / \mathbb{Z}_{2}\right)$ is trivial for nonnegative integer $i$.

Proof. We prove this by induction on $n$.

For $n=1$, it is evident to see that $T^{2} / \mathbb{Z}_{2}$ is homeomorphic to sphere $S^{2}$, hence the proposition follows. Then suppose it is true for $T^{n} / \mathbb{Z}_{2}$. We derive an exact sequence from $(\star)$

$$
0 \rightarrow \tilde{H}_{2 i+1}\left(T^{n+1} / \mathbb{Z}_{2}\right) \rightarrow \tilde{H}_{2 i}\left(T^{n}\right) \stackrel{\left(q_{*}, q_{*}\right)}{\longrightarrow} \cdots
$$

Proposition 4.3 shows that $\operatorname{Ker} q_{*}=0$. Therefore,

$$
\tilde{H}_{2 i+1}\left(T^{n+1} / \mathbb{Z}_{2}\right) \cong \operatorname{Ker}\left(q_{*}, q_{*}\right)=0
$$

The induction is complete.

The homology in even dimension is more complicated. Our aim now is to show the following proposition.

Proposition 5.2 The homology $H_{2 i}\left(T^{n} / \mathbb{Z}_{2}\right)$ has no odd torsion nor higher 2-torsion for $2 \leq 2 i \leq n$.

Proof. Set $X=T^{n}$ and $G=\mathbb{Z}_{2}=\{e, t\}$. We would like to study the structure of $H_{2 i}(X / G)$. Denote by $V$ the 0 -skeleton of $X$. Then $G$ acts trivially on $V$. Thus we consider $V=V / G$ as a subspace of $X / G$. By the long exact sequence of the pair $(X / G, V)$, it is immediate that 


$$
H_{2 i}(X / G) \cong H_{2 i}(X / G, V)
$$

Since $G$ acts freely on $X-V$,

$$
H_{2 i}^{G}(X, V) \cong H_{2 i}(X / G, V)
$$

where $H_{*}^{G}$ means $G$-equivariant homology (cf. [1, Section VII.7]). Recall the long exact sequence of equivariant homology of the pair $(X, V)$ (cf. Section 7 of $[2])$

$$
\cdots \rightarrow H_{k}^{G}(V) \rightarrow H_{k}^{G}(X) \rightarrow H_{k}^{G}(X, V) \rightarrow H_{k-1}^{G}(V) \rightarrow \cdots
$$

To decide $H_{2 i}^{G}(X, V)$, we have to know $H_{*}^{G}(V)$ and $H_{*}^{G}(X)$. For $H_{*}^{G}(X)$, there is a spectral sequence (cf. [1, Section VII.7])

$$
E_{p q}^{2}(X)=H_{p}\left(G ; H_{q}(X)\right) \Rightarrow H_{p+q}^{G}(X)
$$

We compute $H_{p}\left(G ; H_{q}(X)\right)$ now, where $H_{q}(X)$ is a $G$-module. Note that the generator $t$ of $G$ acts on $H_{q}(X)$ as multiplication by $(-1)^{q}$, in fact it is a consequence of the fact that $t$ acts on $H_{1}\left(S^{1}\right)$ as multiplication by -1 together with Künneth formula.

Set $N=t+e$. Note that $N g m=N m\left(g \in G, m \in H_{q}(X)\right)$ and $N H_{q}(X) \subseteq H_{q}(X)^{G}$. Then $N$ induces a map

$$
\bar{N}: H_{q}(X)_{G} \rightarrow H_{q}(X)^{G}
$$

where $H_{q}(X)_{G}$ and $H_{q}(X)^{G}$ denote the group of co-invariants and the group of invariants of $H_{q}(X)$ respectively. We conclude (cf. [1, Section III.1, Example 2])

$$
H_{p}\left(G ; H_{q}(X)\right) \cong \begin{cases}H_{q}(X)_{G}, & p=0 \\ \operatorname{Coker} \bar{N}, & p \geq 1 \text { odd } \\ \operatorname{Ker} \bar{N}, & p \geq 2 \text { even }\end{cases}
$$

To be precise,

- If $q$ is even. The group $G$ acts trivially on $H_{q}(X)$, hence by definition, both $H_{q}(X)_{G}$ and $H_{q}(X)^{G}$ are $H_{q}(X)$ itself. 


$$
\bar{N}: H_{q}(X)=H_{q}(X)_{G} \stackrel{t+e}{\longrightarrow} H_{q}(X)^{G}=H_{q}(X)
$$

is multiplication by 2 . Thus

$$
H_{p}\left(G ; H_{q}(X)\right) \cong \begin{cases}\mathbb{Z}_{\left(\begin{array}{l}
n \\
q
\end{array}\right),} & p=0 \\
\mathbb{Z}_{2}^{\left(\begin{array}{c}
n \\
q
\end{array}\right)}, & p \geq 1 \text { odd } \\
0, & p \geq 2 \text { even }\end{cases}
$$

- If $q$ is odd. The co-invariants $H_{q}(X)_{G}$ is the quotient of $\left.H_{q}(X) \cong \mathbb{Z}^{(n} q\right)$ with respect to its submodule generated by twice of its each element, then $H_{q}(X)_{G} \cong \mathbb{Z}_{2}^{\left(\begin{array}{c}n \\ q\end{array}\right)}$. On the other hand, nothing of $H_{q}(X)$ is fixed by $t \in G$ except 0 , hence $H_{q}(X)^{G}=0$.

$$
\bar{N}: \mathbb{Z}_{2}^{\left(\begin{array}{l}
n \\
q
\end{array}\right)} \cong H_{q}(X)_{G} \stackrel{t+e}{\longrightarrow} H_{q}(X)^{G}=0
$$

is the 0-map. Thus

$$
H_{p}\left(G ; H_{q}(X)\right) \cong \begin{cases}\mathbb{Z}_{2}^{\left(\begin{array}{l}
n \\
q
\end{array}\right)}, & p \geq 0 \text { even } \\
0, & \text { otherwise }\end{cases}
$$

We summarize the results as follows.

$$
\begin{aligned}
E_{p q}^{2}(X) & =H_{p}\left(G ; H_{q}(X)\right) \\
& \cong \begin{cases}\mathbb{Z}^{\left(\begin{array}{c}
n \\
q
\end{array}\right)}, & (p, q)=(0,2 j), j \geq 0 ; \\
\mathbb{Z}_{2}^{\left(\begin{array}{c}
n \\
q
\end{array}\right)}, & (p, q)=(2 i, 2 j+1) \text { or }(2 i+1,2 j), i, j \geq 0 ; \\
0, & \text { otherwise. }\end{cases}
\end{aligned}
$$

One easily checks that the spectral sequence collapses at the $E^{2}$-page. Hence we have computed

$$
H_{2 i}^{G}(X) \cong E_{0,2 i}^{\infty}(X) \cong E_{0,2 i}^{2}(X) \cong \mathbb{Z}^{\left(\begin{array}{c}
n \\
(2 i
\end{array}\right)}
$$

Now for $V$, we have 


$$
H_{k}^{G}(V) \cong H_{k}(G) \oplus \# V \cong \begin{cases}\mathbb{Z}^{2^{n}}, & k=0 \\ \mathbb{Z}_{2}^{2^{n}}, & k \geq 1 \text { odd } \\ 0, & \text { otherwise }\end{cases}
$$

Recall the long exact sequence of the equivariant homology of the pair $(X, V)$

$$
\cdots \rightarrow H_{k}^{G}(V) \rightarrow H_{k}^{G}(X) \rightarrow H_{k}^{G}(X, V) \stackrel{\partial_{k}}{\rightarrow} H_{k-1}^{G}(V) \rightarrow \cdots
$$

For $k=2 i \geq 2$, we derive the following short exact sequence

$$
0 \rightarrow \mathbb{Z}^{\left(\begin{array}{c}
n \\
2 i
\end{array}\right)} \rightarrow H_{2 i}^{G}(X, V) \rightarrow \mathbb{Z}_{2}^{\mu_{i}} \rightarrow 0
$$

for some integer $0 \leq \mu_{i} \leq 2^{n}$ with $\operatorname{Im} \partial_{2 i} \cong \mathbb{Z}_{2}^{\mu_{i}}$, being a subgroup of $H_{2 i-1}^{G}(V) \cong \mathbb{Z}_{2}^{2^{n}}$. We see that $H_{2 i}^{G}(X, V)$ has no odd torsion nor higher 2-torsion.

Finally, together with Theorem 1.1, we complete the proof of Theorem 1.3 by showing the following theorem.

Theorem 5.3 (Conjecture 4.4 of [4]) The integral homology of $T^{n+1} / \mathbb{Z}_{2}$ is of the form

$$
\begin{array}{rlrl}
\tilde{H}_{2 i}\left(T^{n+1} / \mathbb{Z}_{2}\right) \cong \mathbb{Z}_{2}^{a(i, n+1)} \oplus \mathbb{Z}^{b(i, n+1)}, & & 2 \leq 2 i \leq n+1, \\
\tilde{H}_{j}\left(T^{n+1} / \mathbb{Z}_{2}\right) & =0, & & \text { otherwise. }
\end{array}
$$

where

$$
\begin{aligned}
a(i, n+1) & =\tilde{\beta}_{2 i+1}\left(T^{n+1} / \mathbb{Z}_{2} ; \mathbb{Z}_{2}\right), \\
a(i, n+1)+b(i, n+1) & =\tilde{\beta}_{2 i}\left(T^{n+1} / \mathbb{Z}_{2} ; \mathbb{Z}_{2}\right) .
\end{aligned}
$$

Proof. By Proposition 5.1 and Proposition 5.2, it suffices to show that the two equations hold. They are obtained from the universal coefficient theorem. For $2 \leq 2 i \leq n+1$,

$$
\begin{aligned}
\tilde{H}_{2 i}\left(T^{n+1} / \mathbb{Z}_{2} ; \mathbb{Z}_{2}\right) & \cong \tilde{H}_{2 i}\left(T^{n+1} / \mathbb{Z}_{2}\right) \otimes \mathbb{Z}_{2} \\
\tilde{H}_{2 i+1}\left(T^{n+1} / \mathbb{Z}_{2} ; \mathbb{Z}_{2}\right) & \cong \operatorname{Tor}\left(\tilde{H}_{2 i}\left(T^{n+1} / \mathbb{Z}_{2}\right), \mathbb{Z}_{2}\right)
\end{aligned}
$$


These two isomorphisms imply (2) and (1) respectively.

\section{References}

[ 1 ] Brown K. S., Cohomology of Groups. Springer-Verlag New York Inc, New York, 1982.

[ 2 ] Eilenberg S., Homology of Spaces with Operators. I. Trans. Amer. Math. Soc. 61 (1947), 378-417.

[ 3 ] Kozlov D. N., Moduli Spaces of Metric Graphs of Genus 1 with Marks on Vertices. Topology Appl. 156 (2008), 433-437.

[4] Kozlov D. N., The Topology of Moduli Spaces of Tropical Curves with Marked Points. The Asian Journal of Mathematics 13 (2009), 385-404.

[5] Kozlov D. N., Moduli Spaces of Tropical Curves of Higher Genus with Marked Points and Homotopy Colimits. Israel Journal of Mathematics 182 (2011), 253-291.

[ 6 ] Mikhalkin G., Moduli Spaces of Rational Tropical Curves. Proceedings of Gökova Geometry-Topology Conference, Gökova Geometry/Topology Conference (GGT), Gökova, (2006), pp. 39-51.

[ 7 ] Mikhalkin G., Tropical Geometry and Its Applications. International congress of Mathematicians, Vol. II, Eur. Math. Soc., Zürich, (2006), pp. 827-852.

Graduate School of Science

Hokkaido University

Kita Ku, Sapporo 060-0810, Japan

E-mail: liu@math.sci.hokudai.ac.jp 